\title{
Recorrência e migração sísmicas em Montes Claros - MG
}

\author{
Lucas Vieira Barros ${ }^{1}$, Juraci Mario de Carvalho ${ }^{1}$, Diogo Farrapo de Albuquerque ${ }^{1}$, Mônica G. Von Huelsen ${ }^{1}$, Daniel F. \\ Caxeta $^{1}$, Vinicius Martins Ferreira ${ }^{1}$ e Darlan Portela Fontenele ${ }^{1}$ \\ 1 Observatório Sismológico - Universidade de Brasília \\ Brasília - DF, Brasil - lucas@unb.br
}

Copyright 2014, SBGf - Sociedade Brasileira de Geofísica

Este texto foi preparado para a apresentação no VI Simpósio Brasileiro de Geofísica, Porto Alegre, 14 a 16 de outubro de 2014. Seu conteúdo foi revisado pelo Comitê Técnico do VI SimBGt, mas não necessariamente representa a opinião da SBGf ou de seus associados. É proibida a reprodução total ou parcial deste material para propósitos comerciais sem prévia autorização da SBGt.

\section{Resumo}

Este trabalho apresentou os resultados das análises dos dados recentes da rede sísmica de Montes Claros. Observou-se que nos primeiros meses de 2014 (até 11 de abril) houve uma reativação da sismicidade com uma migração dos epicentros para o noroeste, se afastando da cidade. Isto indica uma maior extensão da falha sismogênica. A inversão de formas de ondas em duas estações da rede indica que os regimes de esforços para sismicidade atual e aquela observada até junho de 2013 são semelhantes.

\section{Abstract}

This paper presented the results of the analysis of recent data from the seismic network of Montes Claros. It was observed that in early 2014 (until April 11th) there was a reactivation and migration of the Montes Claros seismicity with epicenters moving to the northwest, away from the city. This indicates a greater extent of seismogenic fault. The inversion of waveforms at two stations of the network indicates that the stress regimes of the current seismicity and those observed by June 2013 are similar.

\section{Introdução}

A sismicidade recente em Montes Claros - MG iniciouse em 05 de março de 2011, às 23h29 (GMT), com um sismo de magnitude 3,2 na Escala Richter sentido por várias pessoas da zona urbana da cidade. Antes a terra já havia tremido em 2009 (1/2, M1,3, 2/2, M2,4, 1/8, M2,1 e 3/8, M1,9) e 2010 (29/9, M2,8 e M2,4). Estes sismos foram todos sentidos pela população de Montes Claros. Entretanto, somente em 2012 aconteceram os maiores eventos. O evento principal, em 19/05/2012, às 15:49:35, magnitude 4,0 mR, sentido com intensidade V-VI (MM ) e dois eventos no dia 19/12, com magnitude de 3,6 mR, às 04:54:39 e 05:31:17, respectivamente. Entretanto se tem notícias de sismos sentido em Montes Claros em 1978 (Berrocal et al. 1984), 1995, 1996 e 1999. Após o evento principal de 19/05/2012, as universidades de São Paulo e de Brasília instalaram uma rede sismográfica com nove estações para estudar a atividade de réplicas.

Os dados produzidos pela rede sismográfica de Montes
Claros desde sua instalação, em maio de 2012, até junho de 2013 foram analisados e os resultados publicados por Farrapo et al.(2013), Assumpção (2013), Assumpção et al.(2013) e Hans et al.(2014). Este último é mais conclusivo e indica que os tremores de 2012 e 2013 ocorreram numa falha geológica, até então desconhecida, de orientação NNW-SSE e mergulho para leste. Estudos de mecanismo focal composto e por inversão de formas de ondas indicam que as tensões na área têm orientação E-W. A inversão de vários mecanismos focais de sismos na parte norte de Minas Gerais indicam que a movimentação nesta falha, bem como em outras áreas vizinhas resulta de tensões compressivas aproximadamente E-W que atuam em toda a crosta terrestre nesta parte norte de Minas Gerais (Hans et al. 2014).

Após junho de 2013 permaneceram em operação duas estações da Universidade de Brasília (UnB) (MC03 e MC04) e, a partir de janeiro de 2014, mais uma estação MC01, pertencente à Universidade de Montes Claros (Unimontes). As análises dos dados produzidos nos últimos seis meses, até abril de 2014 indicam uma recorrência sísmica a partir de primeiro de abril, quando aconteceu um evento de magnitude 3,0 e em 06/04 dois eventos de magnitudes de 3,3, sentidos com intensidade (MM) V.

Essa reativação sísmica motivou uma viagem a Montes Claros para esclarecimentos à população e instalação de mais duas estações, perfazendo uma rede com cinco estações, que entrou em operação a partir de 12 de abril (Barros, 2014).

A atividade sísmica recente observada em Montes Claros, no mês de abril/2014 ocorreu na mesma falha definida por Hans et al. (2014). Entretanto, resultou de mobilizações em uma parte ainda não conhecida. Os sismos migraram mais para o norte, se afastando da cidade. Estudos de mecanismo focal, por inversão de forma de ondas, com os eventos recentes, indicam que o regime de esforço é o mesmo para o caso passado e que os parâmetros da falha são mais ou menos concordantes com aqueles definidos previamente.

Este trabalho tem como objetivo apresentar os resultados das análises dos dados produzidos de janeiro a abril de 2014, discutindo essa reativação e migração dos epicentros.

\section{A nova rede sismográfica de Montes Claros}

A reativação da sismicidade em Montes Claros, com sismos de intensidade até $\mathrm{V}$ (MM), motivou a reinstalação de instrumentos sismográficos na área. Atualmente existe uma rede de cinco estações, 
operando na banda de $0,03 \mathrm{~Hz}$ a $100 \mathrm{~Hz}$, com ampla faixa dinâmica $(+130 \mathrm{~dB})$, com gravação contínua a uma taxa de amostragem de 100 sps (Fig. 1). A estação MC01, localizada na reserva ambiental (parque Lapa Grande), de propriedade da Unimontes, envia os seus dados em tempo real, via internet, os quais estão sendo acessados pelas Universidades de São Paulo, de Brasília e de Montes Claros. Com isso essa sismicidade recente vem sendo monitoradas por essas três instituições.

Existe também a previsão de instalação de mais uma estação, mais próxima dos epicentros, no local onde outrora funcionou a estação MC09. Isto deverá acontecer ainda neste mês de junho.

Com essa rede de seis estações, com a geometria mostrada na Fig.1, os possíveis eventos futuros serão localizados com muito mais precisão e, dessa forma a migração sísmica na área será checada de forma mais segura.

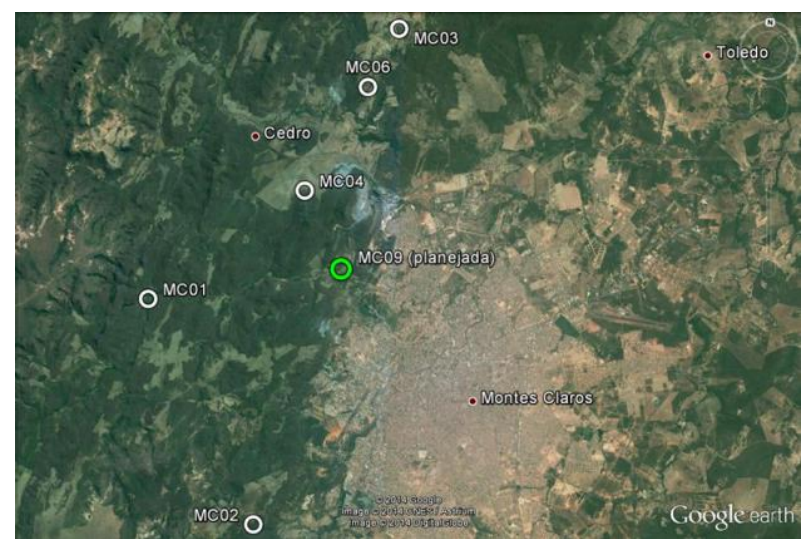

Figura 1 - Mapa com as localizações das cinco estações que estão em operação em Montes Claros (MG) e da estação planejada. A estação MC01 pertence à Unimontes e os seus dados são enviados via internet. As demais pertencem à UnB e tem gravação local.

\section{Análise dos dados recentes}

Os dados coletados nas estações MC01, MC04 e MC03, do período de $1^{\circ}$ de janeiro a 12 de abril de 2014, foram analisados em Brasília. Infelizmente, constatou-se que a estação MC04, por problema no sistema de alimentação elétrico dos instrumentos, não gerou dados em todo o período; a estação MC01 funcionou apenas a partir do dia 21 de fevereiro, quando técnicos da UnB estiveram no local; a estação MC03 funcionou corretamente durante todo o período. Portanto, nos meses de fevereiro, março e parte de abril as estações MC01 e $\mathrm{MC0} 3$ funcionaram simultaneamente e detectaram 52 eventos, sendo 30 eventos naturais (tectônicos) e 22 artificiais (detonações em pedreiras da região de Montes Claros). Para alguns eventos foi difícil diferenciar explosões de sismos naturais, devido à grande semelhança de suas formas de ondas, visto que os epicentros de ambos os tipos de eventos estão muito próximos. Entretanto, mesmo desconhecendo o plano de fogo das pedreiras da região, foi possível verificar que as detonações são feitas em torno de horários fechados, com variação de minutos e sempre na parte da tarde (veja a Figura 2).

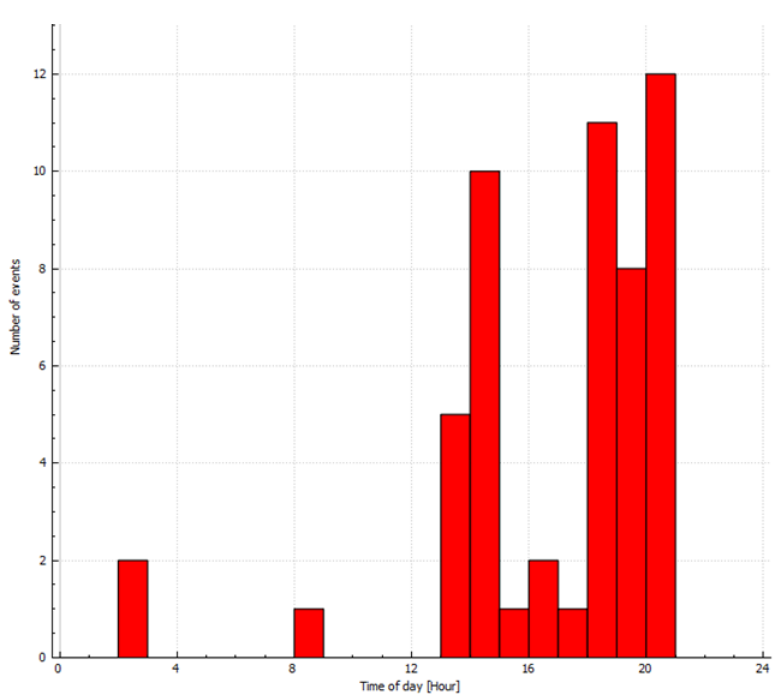

Figura 2 - Distribuição de todos os eventos durante 0 dia. Observa-se uma concentração de eventos entre 18 e 21 horas e entre 14 e 15 horas (GMT), o que corresponde ao horário de realização de detonações.

\section{Localização hipocentral - segunda sequência}

Todos os 52 (cinquenta e dois) eventos foram analisados no SEISAN - the earthquake analysis software (Havskov, J. \& Ottemöller, 2008) e localizados com o programa hypocenter (Lienert,1994). Aqueles que o foram com dados de uma única estação (dezenove eventos) tiveram as suas profundidades fixadas na superfície, visto que os dados de uma estação de três componentes não são suficientes para determinar o hipocentro de um sismo, apenas o epicentro (latitude, longitude e tempo de origem). Neste caso, foram lidas as fases $P, S$ e 0 Azimute, determinado pelas amplitudes da fase $P$ nos três eixos ortogonais (Vertical - Norte-Sul e Leste-Oeste).

Com duas estações foram localizados 33 (trinta e três) eventos, dos quais 11 (onze) eram explosões. Entretanto, considerando a precisão que esse tipo de dado pode dar, foi feita uma nova seleção com os 18 eventos naturais mais bem localizados, com erro de localização horizontal da ordem de $+/-1 \mathrm{~km}$ ou menor (veja Tabela 1) e plotados nos mapas das figuras 3 e 4. As foram feitas com o modelo desenvolvido por (Hans et al; 2014).

\section{Localização hipocentral - primeira sequência}

Para comparar as localizações epicentrais dos sismos recentes com os sismos da primeira sequência foi feita uma seleção dos melhores eventos da primeira sequência (doze eventos) apresentados na Tabela 2. Essa Tabela foi cedida pelo IAG - USP com as localizações feitas usando diferentes técnicas e por diversos pesquisadores da USP. Dessa forma a precisão é da ordem de $1 \mathrm{~km}$ ou menor e as magnitudes podem variar para mais ou para menos 
em 0,3 unidades (Comunicação pessoal, Prof. Marcelo Assumpção).

Tabela 1 - Eventos bem localizados da segunda sequência sísmica em Montes Claros, detectados pela rede local (estações MC01 e MC03) no período de 1 de janeiro a 12 de abril de 2014.

\begin{tabular}{|c|c|c|c|c|c|c|c|c|c|c|c|c|}
\hline No & $\begin{array}{c}\text { Data } \\
\text { (dd/mm/aaaa) }\end{array}$ & $\begin{array}{c}\text { Tempo } \\
\text { origem (UT) } \\
\text { (hh:mm:ss.ss) }\end{array}$ & $\begin{array}{c}\text { Lat } \\
\text { (graus) }\end{array}$ & $\begin{array}{c}\text { Long } \\
\text { (graus) }\end{array}$ & $\begin{array}{c}\text { Prof } \\
\text { (Km) }\end{array}$ & $\begin{array}{c}\text { Mag } \\
\mathbf{m D}\end{array}$ & No & $\begin{array}{c}\text { Gap } \\
\text { (Graus) }\end{array}$ & $\begin{array}{c}\text { Dmin } \\
\text { (Km) }\end{array}$ & $\begin{array}{c}\text { Rms } \\
\text { (s) }\end{array}$ & $\begin{array}{c}\text { Erh } \\
\text { (km) }\end{array}$ & $\begin{array}{c}\text { Erx } \\
(\mathbf{k m})\end{array}$ \\
\hline 1 & $21 / 02 / 14$ & $19: 58: 04.47$ & -16.692 & -43.895 & 1.0 & 0.6 & 5.0 & 237 & 5.3 & 0.01 & 1.0 & 1.0 \\
\hline 2 & $24 / 02 / 14$ & $02: 25: 37.13$ & -16.705 & -43.892 & 3.5 & 0.1 & 6.0 & 259 & 5.3 & 0.02 & 0.2 & 0.2 \\
\hline 3 & $17 / 03 / 14$ & $18: 15: 23.70$ & -16.684 & -43.903 & 0.1 & 0.8 & 6.0 & 214 & 5.5 & 0.06 & 0.6 & 0.4 \\
\hline 4 & $01 / 04 / 14$ & $16: 07: 01.05$ & -16.692 & -43.876 & 1.9 & 1.3 & 6.0 & 262 & 5.9 & 0.07 & 0.4 & 0.2 \\
\hline 5 & $01 / 04 / 14$ & $16: 11: 21.89$ & -16.684 & -43.896 & 1.0 & 2.0 & 6.0 & 225 & 5.2 & 0.01 & 0.3 & 0.2 \\
\hline 6 & $02 / 04 / 14$ & $20: 44: 37.96$ & -16.673 & -43.910 & 0.1 & 1.6 & 5.0 & 184 & 4.8 & 0.01 & 0.7 & 0.5 \\
\hline 7 & $06 / 04 / 14$ & $13: 39: 29.57$ & -16.687 & -43.893 & 0.8 & 3.3 & 6.0 & 234 & 5.4 & 0.02 & 0.2 & 0.2 \\
\hline 8 & $06 / 04 / 14$ & $13: 43: 51.19$ & -16.696 & -43.885 & 0.7 & 0.7 & 5.0 & 255 & 6.3 & 0.03 & 1.2 & 0.2 \\
\hline 9 & $06 / 04 / 14$ & $13: 44: 20.34$ & -16.682 & -43.899 & 0.7 & 0.1 & 5.0 & 217 & 5.1 & 0.01 & 0.8 & 0.7 \\
\hline 10 & $06 / 04 / 14$ & $14: 15: 46.97$ & -16.687 & -43.897 & 1.4 & 0.9 & 6.0 & 227 & 5.6 & 0.04 & 0.3 & 0.2 \\
\hline 11 & $06 / 04 / 14$ & $14: 47: 00.86$ & -16.697 & -43.880 & 0.8 & 2.0 & 5.0 & 261 & 6.4 & 0.04 & 1.0 & 1.0 \\
\hline 12 & $06 / 04 / 14$ & $14: 48: 20.63$ & -16.690 & -43.889 & 0.7 & 0.6 & 5.0 & 243 & 5.7 & 0.02 & 1.0 & 0.9 \\
\hline 13 & $06 / 04 / 14$ & $14: 50: 20.65$ & -16.685 & -43.896 & 0.7 & 0.0 & 5.0 & 227 & 5.3 & 0.01 & 0.8 & 0.7 \\
\hline 14 & $06 / 04 / 14$ & $14: 51: 15.58$ & -16.682 & -43.897 & 0.7 & 0.1 & 5.0 & 219 & 5.0 & 0.01 & 0.8 & 0.7 \\
\hline 15 & $06 / 04 / 14$ & $14: 56: 36.93$ & -16.679 & -43.900 & 0.0 & 0.7 & 5.0 & 210 & 4.9 & 0.02 & 0.7 & 0.3 \\
\hline 16 & $06 / 04 / 14$ & $19: 31: 05.22$ & -16.687 & -43.892 & 0.8 & 3.3 & 5.0 & 236 & 5.4 & 0.01 & 0.9 & 0.8 \\
\hline 17 & $06 / 04 / 14$ & $19: 34: 46.99$ & -16.680 & -43.899 & 1.0 & 1.8 & 5.0 & 213 & 4.9 & 0.01 & 0.8 & 0.3 \\
\hline 18 & $08 / 04 / 14$ & $02: 55: 24.79$ & -16.681 & -43.894 & 0.7 & 2.9 & 5.0 & 224 & 4.9 & 0.01 & 0.8 & 0.2 \\
\hline
\end{tabular}

Tabela 2: - Eventos bem localizados da primeira sequencia sísmica em Montes Claros/MG. Essas localizações foram feitas por diferentes pesquisadores do Centro de Sismologia da Universidade de São Paulo, usando diferentes técnicas e recursos de modo a garantir uma boa precisão.

\begin{tabular}{|c|c|c|c|c|c|c|c|}
\hline No & $\begin{array}{c}\text { Data } \\
\text { (dd/mm/aaaa) }\end{array}$ & $\begin{array}{c}\text { Time Origin } \\
\text { (hh:mm:ss.ss) }\end{array}$ & $\begin{array}{c}\text { Lat } \\
\text { (DD) }\end{array}$ & $\begin{array}{c}\text { Long } \\
\text { (DD) }\end{array}$ & $\begin{array}{c}\text { Prof } \\
\text { (Km) }\end{array}$ & Mag & Io \\
\hline 1 & $05 / 03 / 2011$ & $23: 28: 45.4$ & -16.701 & -43.883 & 1.0 & 3.3 & - \\
2 & $19 / 05 / 2012$ & $13: 41: 22.56$ & -16.696 & -43.883 & 1.1 & 4.0 & $\mathrm{~V}-\mathrm{VI}$ \\
3 & $12 / 09 / 2012$ & $23: 56: 45.68$ & -16.704 & -43.885 & 1.2 & 2.9 & - \\
4 & $19 / 12 / 2012$ & $04: 54: 38.49$ & -16.697 & -43.879 & 1.4 & 3.5 & - \\
5 & $19 / 12 / 2012$ & $05: 31: 16.86$ & -16.700 & -43.882 & 1.3 & 3.5 & - \\
6 & $18 / 04 / 2013$ & $10: 10: 52$ & -16.697 & -43.889 & 1.5 & 3.5 & - \\
7 & $06 / 04 / 2014$ & $13: 39: 29.38$ & -16.692 & -43.898 & 0.8 & 3.3 & $\mathrm{~V}$ \\
8 & $06 / 04 / 2014$ & $19: 31: 05.12$ & -16.687 & -43.893 & 1.2 & 3.3 & $\mathrm{~V}$ \\
\hline
\end{tabular}




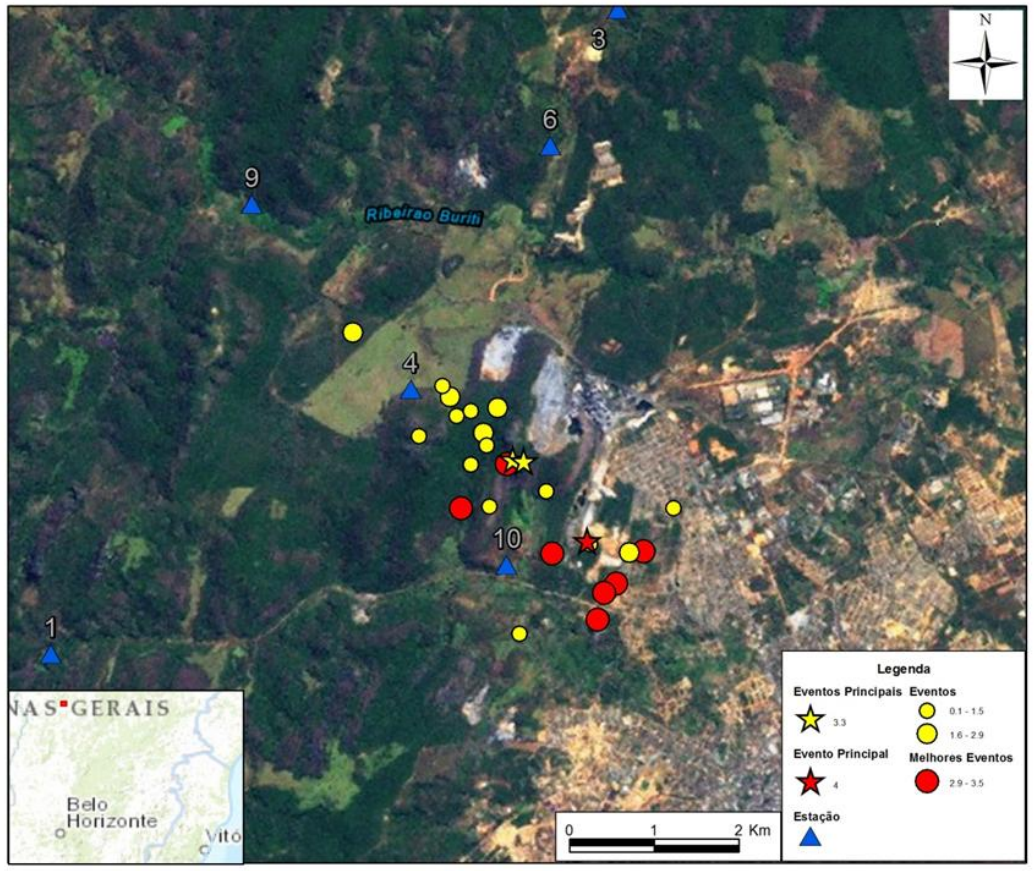

Figura. 3 -- Distribuições da sismicidade de 2011-2013 (círculos vermelhos) e da sismicidade recente (2014) (círculos amarelos) em Montes Claros - MG. Para a sismicidade de 2011-2013 apenas foram plotados os eventos com as melhores localizações. As estrelas denotam os maiores sismos das duas sequências. A estrela vermelha indica a localização do sismo principal de 19 de maio de 2012, magnitude 4.0mR. As duas estrelas amarelas indicam os epicentros dos dois sismos de 6 de abril recente, acontecidos às $10 \mathrm{~h} 39 \mathrm{~m}$ e $16 \mathrm{~h} 31 \mathrm{~m}(\mathrm{H}$. Local), respectivamente. Os triângulos azuis denotam estações sismográficas. Os dois eventos mais a NW (círculos vermelhos) são os eventos de 6 de abril de 2014, localizados pelo Centro de Sismologia do IAG-USP, com estações regionais, usando correlação de forma de ondas.

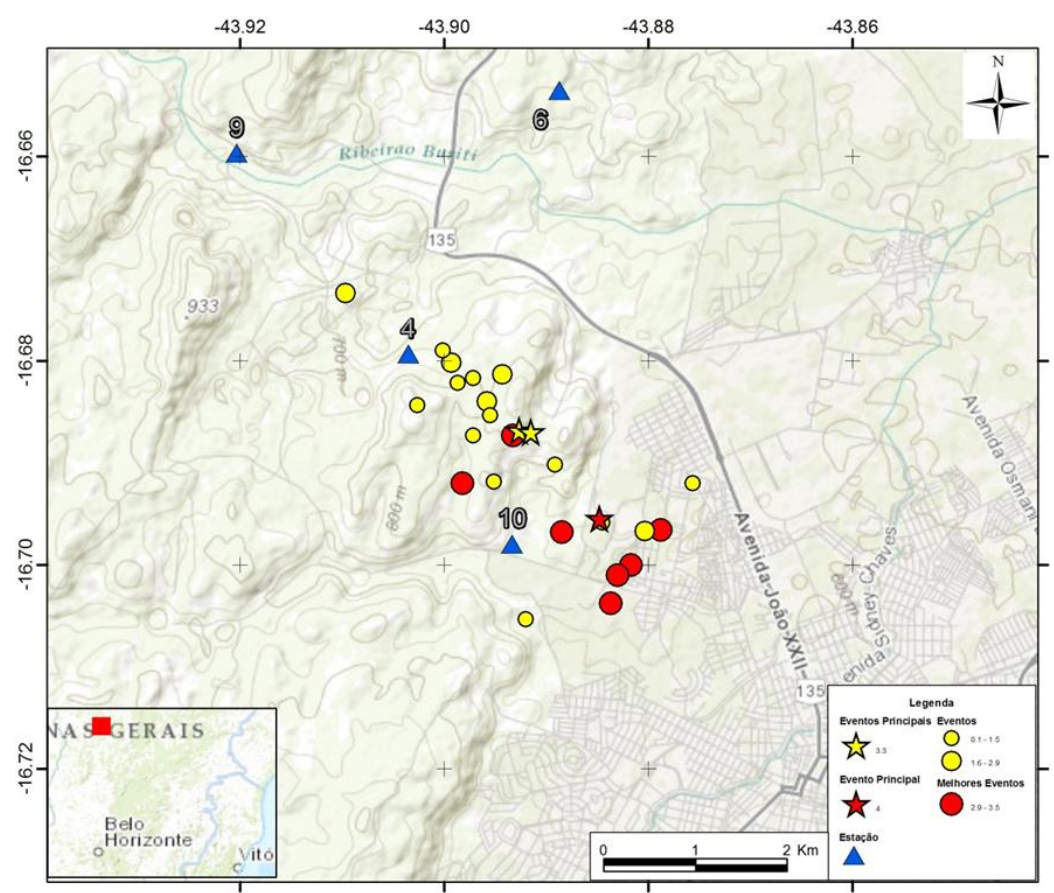

Figura 4 - Mapa topográfico com a distribuição da sismicidade de 2011-2013 (círculos vermelhos) e da sismicidade recente (2014) (círculos amarelos) em Montes Claros - MG. As estrelas denotam os maiores sismos das duas sequências. A estrela vermelha indica a localização do sismo principal de 19 de maio de 2012. As duas estrelas amarelas indicam os epicentros dos dois sismos de 6 de abril recente, acontecidos às $10 \mathrm{~h} 39 \mathrm{~m}$ e $16 \mathrm{~h} 31 \mathrm{~m}$, respectivamente. Os triângulos azuis denotam estações sismográficas. Os dois eventos mais a NW (círculos vermelhos) são os eventos de 6 de abril de 2014, localizados pelo IAG-USP, com estações regionais por correlação de forma de ondas. 


\section{Inversão de formas de ondas}

A inversão foi realizada usando o código ISOLA (Zahradnik et al, 2005; Sokos and Zahradinik 2008), o MATLAB e um método iterativo, similar aquele desenvolvido por Kikuchi Kanamori (1991). As funções de Green foram determinadas usando o Modelo 1-D de Hans et. al.(2014) e o método do número de onda discreto desenvolvido por Bouchon (1981).

Foram utilizados dados de um único evento (evento 17 da Tabela 1) registrado por apenas duas estações (MC01 e MC03). Após a remoção do efeito instrumental, as formas de onda foram filtradas na banda de $0.5 \mathrm{~Hz}$ a $1.0 \mathrm{~Hz}$, passando para o programa ISOLA as seguintes informações relativas ao evento (evento 17): epicentro, tempo de origem, profundidade e magnitude. Adicionalmente também foram passadas as polaridades do evento nas duas estações (MC01 e MC04). O processo consistiu em, iterativamente procurar em uma grade a melhor profundidade e 0 tempo de centróide de modo que a correlação entre as formas de ondas reais e sintéticas fosse máxima, ou seja, os sinais de entrada e os sintéticos sejam os mais semelhantes possíveis. Quando atingido esse ponto de busca os parâmetros da fonte correspondem aos parâmetros da fonte verdadeira e daí se completa o processo de inversão.

\section{Resultados}

As figuras 3 e 4 mostram a distribuição da sismicidade recente em Montes Claros (circulos amarelos) e da sismicidade passada (circulos vermelhos). As estrelas representam as localizações dos eventos principais das duas sequencias: estrela vermelha evento principal de $19 / 05 / 2012$ e as duas estrelas amarelas são os dois eventos de 06/04/2014, magnitudes 3,3 mR. Os eventos na cor amarelo foram localizados neste trabalho e na cor vermelho foram localizados por diferentes pesquisadores do Centro de Sismologia (CS) da Universidade de São Paulo (USP), usando diferentes técnicas. Os dois círculos vermelhos mais a NW são os epicentros dos dois eventos 06/04/2014, determinados, por correlação de forma de onda, pelo CS-USP. Observe que um desses eventos tem epicentro igual ao determinado nesse trabalho. Os outros dois diferem em mais ou menos $1 \mathrm{~km}$.

A Fig. 5 apresenta os resultados da inversão de formas de onda do evento de 06/04/2014, às 19:34:46.99 (GMT) registrado pelas estações MC01 e MC04. O evento foi localizado à meia distância entre MC1 e MC3. A Fig. 6 mostra as formas de ondas sintéticas (vermelha) e observadas (preto) para os dois eventos em todas as componentes de movimento do chão.
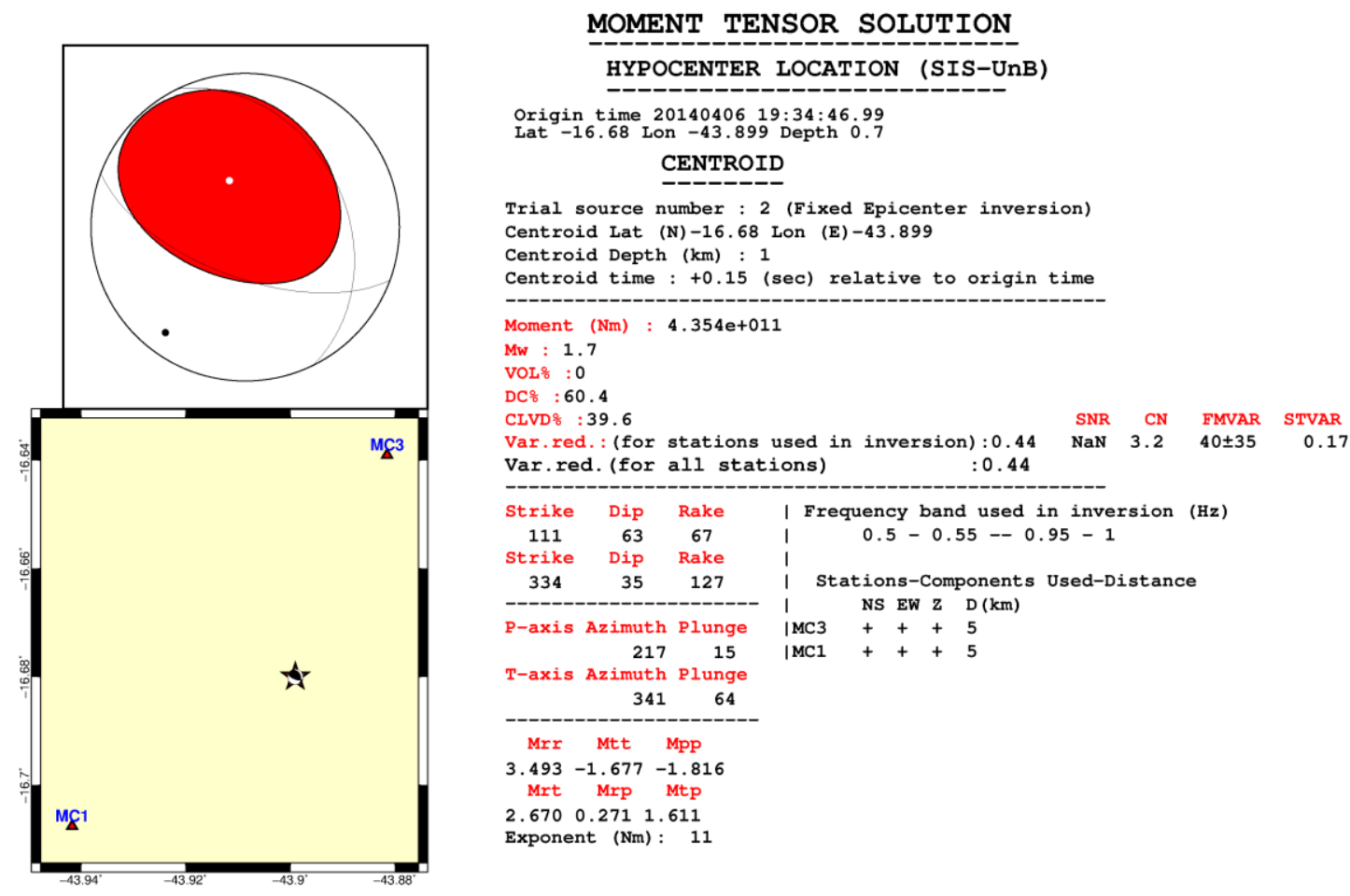

Fig. 5: - Resultados da inversão de formas de onda do evento de 06/04/2014, às 19:34:46.99 (GMT) registrado pelas estações MC01 e MC04. O evento está à meia distância entre MC01 e MC03. 


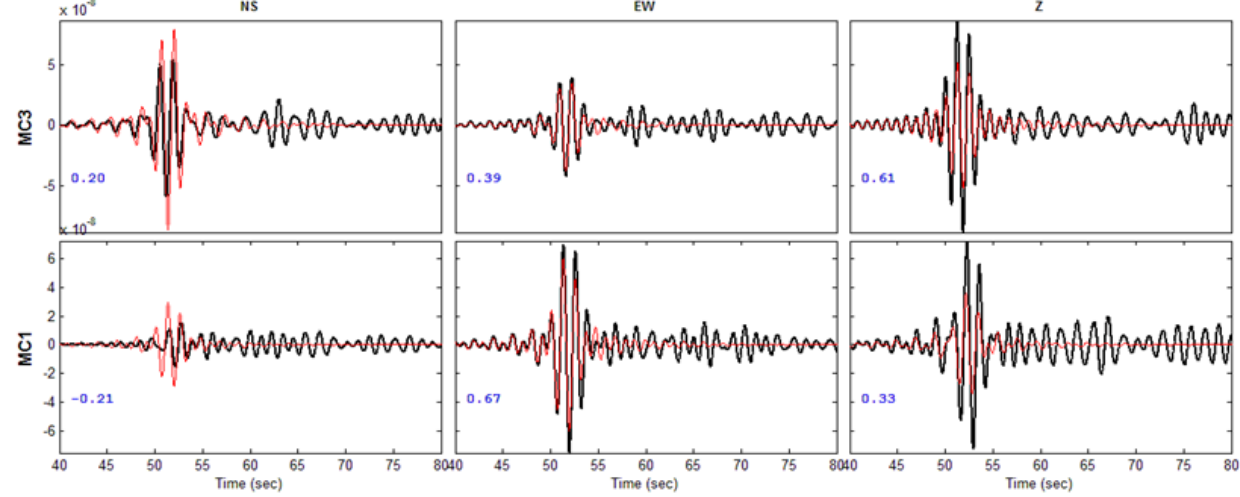

Fig. 6: - Formas de ondas sintéticas (vermelhas) e observadas (pretas) nas estações MC01 e MC03 para o sismo de 06/04/2014.

\section{Discussão e Conclusões}

Este trabalho apresenta os resultados da análise e interpretação dos dados gerados por duas estações sismográficas instaladas em Montes Claros - MG, entre janeiro e abril de 2014. Podemos concluir preliminarmente que a sismicidade recente em abril de 2014 resultou da ativação de um novo segmento da falha sismogênica de Montes Claros; onde ainda não havia sido observada sismicidade. Como mostram as figuras 3 e 4 , os sismos migraram mais para NW (círculos amarelos), em direção oposta à cidade, se distanciando. Em contrapartida, estão mostrando que a falha ativa tem uma dimensão maior do que aquela previamente determinada por Hans et. al (2014) a partir da sismicidade de 2011-2013. Sem considerar os erros de localização a falha se estendeu mais ou dois quilômetros para noroeste.

Duas conclusões podem ser tiradas: 1 . Os sismos, ao se distanciarem, terão os seus efeitos reduzidos, e causarão menor intensidade na cidade; 2.Esse distanciamento, no entanto, implica maior dimensão da falha, que poderia ter potencial para gerar um sismo ligeiramente maior que o de maior magnitude já observada. Entretanto, existem casos, inclusive no Brasil, em que falha muito extensa, resultante de migração sísmica e com potencial para gerar sismo de alta magnitude, nunca se mobilizou em sua totalidade. Este é o caso da falha de Samambaia em João Câmara no Rio Grande do Norte (Ferreira et al., 1987; Takeya et. al, 1989).

A inversão apesar de ter sido feita para um único evento nas três componentes de duas estações produziu resultados satisfatórios (Fig. 7), se compararmos com aqueles resultados do mecanismo focal composto determinado por Hans et. al (2014). Isto nos diz que a falha é comum às duas sismicidades.
O mecanismo focal, a despeito de resultar da inversão em apenas seis componentes de duas estações apresentou parâmetros de qualidade da inversão, que, apesar de não serem ótimos, tem valores aceitáveis de porcentagem double-couple (DC\%), redução de variância (VR), relação sinal ruído (SNR), bem como Polaridade consistentes e magnitudes de duração e Mw compatíveis, conforme se pode observar na Fig.5.

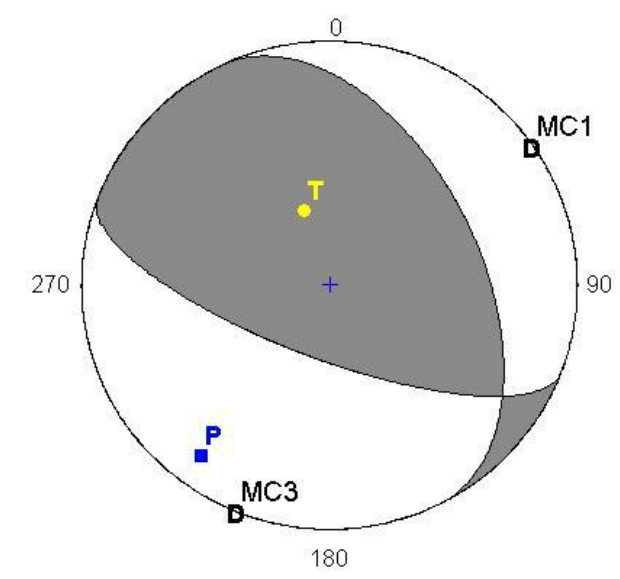

Figura 7: - Mecanismo focal resultante da inversão de formas de onda do evento de 06/04/2014 nas estações MC1 e MC3. U representa as polaridades nas componentes verticais da primeira chegada da onda $P$ em cada estação. Observe a Consistência da localização dessas polaridades no mecanismo focal resultantes da inversão. Strike (11/344) dip (63/35) e rake (67/127). Azimute e plunge do eixo P 217 e 15: azimute do e plunge do eixo T (341 e 64). 


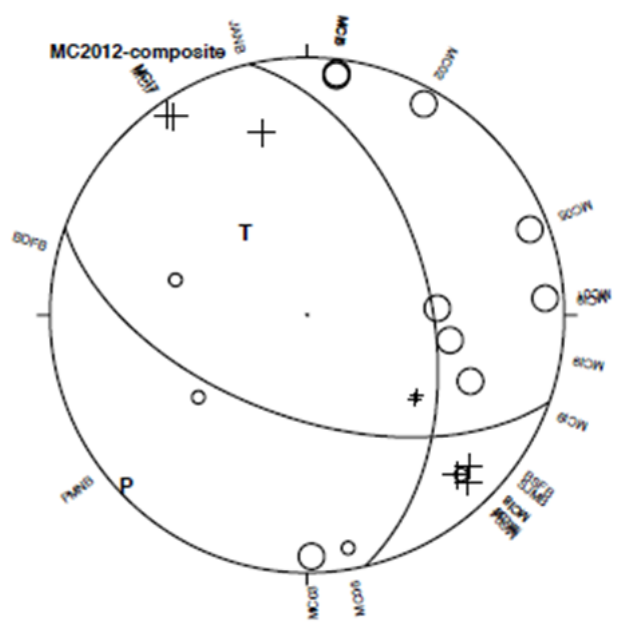

Fig. 8. Mecanismo focal composto para a sismicidade de Montes Claros 2012-2013. Plano de falha: strike, dip, rake (principal/auxiliar):110/347, 57/50 e 50/135.

Conclusivamente os resultados obtidos neste trabalho são compatíveis com aqueles obtidos por Hans et. al. (2014), como pode ser visto nas figuras 7 e 8 . Ambos com falhamento inverso, com parâmetros de falhas muito semelhantes.

\section{Referências}

Albuquerque, D.F., Assumpção, M., Franca, G.S., Von Huelsen, M.G., Barbosa, J.R. and Galhardo, L., 2012. Estudos preliminares da sismicidade registrada em Montes Claros, Minas Gerais. Expanded abstract, V Simpósio Brasileiro de Geofísica, Salvador, Brazil, 27-29 November 2012.

Assumpção, M, 2013. Estudo dos tremores de terra de Montes Claros. Relatório Técnico de 11 de março de 2013. Centro de Sismologia da Universidade de São Paulo, $14 \mathrm{p}$.

Assumpção, M., and Sacek, V., 2013. Intra-plate seismicity and flexural stresses in central Brazil, Geophys. Res. Lett., 40, 487-491, doi:10.1002/grl.50142.

Assumpção, M., Veloso, J.A.V., Barbosa, J.R., Blum, M.L.B., Carvalho, J.M., Neves, E., Bassini, A., 1990. The Manga earthquakes, MG, March of 1990. XXXVI Brazilian Congress of Geology, 1990, Natal-RN. Proceedings of the XXXVI Brazilian Congress of Geology, vol. 6. SBG, NatalRN, pp. 2154-2159.

Assumpção, M., Farrapo, D., Galhardo, L., Moreira, M.F., Huelsen, M., Franca, G.S., Leite, M., Barros, L.V, Silva, F.F. and Barbosa, J.R., 2013. The 2012 Montes Claros earthquake sequence in the Sao Francisco craton: another evidence of inverse faulting and compressional stresses in Eastern Brazil. 13th Int. Congr. of the Brazilian Geophys. Soc., Rio de Janeiro, Brazil, August 26-29, 2013.

Barros, L.V., 2014. Síntese das ações empreendidas pela equipe do Observatório Sismológico da Universidade de Brasília na visita a Montes Claro-
MG, entre os dias 9 e 13 de abril de 2014 . Relatório técnico, 13 p www.obsis.unb.br.

Bouchon, M. (1981). A simple method to calculate Green`s functions for elastic layered media”, Bull. Seis. Soc. Am., 71, 959-971.

Ferreira, J. M. ; Takeya, M. K. ; Costa, J. M. ; Moreira, J. A. M. ; Assumpção, M. ; Veloso, J. A. V. ; Pearce, R. G., 1987. A continuing intraplate earthquakes sequence near João Câmara northeastern Brazil - preliminary results Geophysics Research Letters, v. 14, p. 1042-1045

Hans, A., M. Assumpção, C. Ciardelli, D.F. Albuquerque, L.V. Barros, G.S.L.França, 2014. The 2012-2013 Montes Claros earthquake series in the São Francisco Craton, Brazil: new evidence for non-uniform intraplate stresses in mid-plate South America. Geophys.J.Int., submitted.

Havskov, J. \& Ottemöller, L. (Eds.). SEISAN: The Earthquake Analysis Software, Version 8.1. Inst. of Solid Earth Physics, University of Bergen, Norway, 2008. 227 p.

Kikuchi, M., \& Kanamori., (1991). "Inversion of complex body waves III”, Bull. Seis. Soc. Am. 81, 6, 23352350.

Lienert, B. R. Hypocenter 3.2: A computer Program for Locating Earthquakes Locally, regionally and globally. Hawaii Institute of Geop. \& Planetology, Corea, 1994.

Sokos, E. N. \& J. ZAHRADNÍK, J., (2008). ISOLA a Fortran code and a Matlab GUI to perform multiple- point source inversion of seismic data, Comput. Geosci. 34, 967-977.

Takeya, M. K. ; Ferreira, J. M., Pearce, R. G., Assumpção, M., Sophia, C.M. Costa J.M., 1989. The 1986-1987 intraplate earthquakes sequence near João Câmara, northeast Brazil - evolution of seismicity. Tectonophysics, v. 167, p. 117-131.

Zahradnik, J., and Plesinger, A., 2005. Long-period pulses in broadband records of near earthquakes. Bull. Seism. Soc. Am., 95, 1928-1939.

Zahradnik, J., and S. Custódio (2012). Moment tensor resolvability: Application to southwest Iberia. Bull. Seism. Soc. Am., 102, 1235-1254, doi: 10.1785/0120110216. 